\title{
The impact of intraoperative ultrasonography on the surgical treatment of patients with colorectal liver metastases
}

\author{
C. Sietses $\cdot$ M. R. Meijerink $\cdot$ S. Meijer $\cdot$ \\ M. P. van den Tol
}

Received: 7 August 2009/Accepted: 19 December 2009/Published online: 29 January 2010

(C) The Author(s) 2010. This article is published with open access at Springerlink.com

\begin{abstract}
Introduction Intraoperative ultrasonography (IOUS) has been the standard in surgical decision making in oncologic liver surgery. Preoperative imaging techniques have improved substantially in resent years; therefore, the importance of IOUS might change. The current results of IOUS were compared with preoperative high-resolution helical CT scanning and the impact of IOUS on surgical decision making was evaluated.

Methods A total of 100 consecutive patients who underwent open surgery for colorectal liver metastases within 4 weeks after preoperative imaging, performed with highspeed helical CT scanners, were included for this study. During surgery, IOUS was performed by a liver specialized radiologist. The findings on preoperative and intraoperative imaging and surgical exploration were compared regarding number, site, and size of the hepatic lesions. The preoperative surgical plan was compared with the final surgical treatment.

Results One hundred patients with CRLM underwent 117 surgical treatments. In 38 patients IOUS differed from preoperative data. In 23 cases IOUS identified more metastatic lesions. In five patients, intraoperative findings identified smaller or less hepatic lesions. Additional information on the localization of the hepatic lesions was gathered by IOUS and changed the surgical treatment in ten cases. IOUS alone altered the surgical strategy 35 times
\end{abstract}

C. Sietses · S. Meijer · M. P. van den Tol ( $\varangle)$

Department of Surgery, Vrije Universiteit Medical Centre,

P.O. Box 7057, 1007 MB Amsterdam, The Netherlands

e-mail: mp.vandenTol@vumc.nl

M. R. Meijerink

Department of Radiology, Vrije Universiteit Medical Centre,

Amsterdam, The Netherlands during 117 procedures. In nearly all cases, discrepancy between the preoperative CT scan and IOUS resulted in a change of surgical treatment.

Conclusions Despite improvement in preoperative imaging technology, the intraoperative use of ultrasonography remains of crucial importance. The detection of preoperatively unknown lesions remains high with great consequence on surgical therapy.

Keywords Radiology · Ultrasonography · Colorectal liver metastasis $\cdot$ Liver surgery

Preoperative mapping of metastatic lesions in the liver is of crucial importance when patients are considered for surgical treatment. Computer tomography (CT) and intraoperative ultrasonography (IOUS) are most frequently used. Historically, CT missed approximately $20-40 \%$ of the malignant lesions compared with IOUS. The influence of IOUS on surgical decision making for hepatic malignancies has been documented by numerous reports since the $1980 \mathrm{~s}$. The preoperatively planned surgical procedures were changed as a result of IOUS in 19-65\% of cases [1-4].

CT scanning techniques (as well as ultrasonographic technology) have changed dramatically since then. The accuracy of modern imaging with high-resolution helical CT scanners has increased. However, the exact role of these techniques for the analysis of liver metastasis and for surgical decision making remains unclear.

The goal of this study was to determine the accuracy of preoperative CT scanning compared with IOUS in patients who were treated surgically for colorectal liver metastasis. The preoperative surgical plan was compared with the final surgical treatment to see whether intraoperative findings 
(IOUS and surgical exploration) affected the treatment strategy in our patient population.

\section{Methods}

One hundred consecutive patients who underwent surgery for colorectal liver metastases were included. All patients underwent preoperative helical CT imaging. Patients who are described in this series were for a large part also included in a Dutch national study in which patients were randomized between workup and follow-up with or without FDG-PET. Therefore, not all patients were analyzed with FDG-PET scanning.

In almost all patients referred to our institution, metastatic lesions were identified or suspicion was raised based on (periodic screening) transabdominal ultrasonography of the liver and/or an increase of serum CEA levels.

Routine preoperative 4-phase helical CT scans of the upper abdomen were obtained on a 4-slice multi-detector CT scanner (Somatom Volume Zoom, Siemens, Erlangen, Germany) or a 64-slice multi-detector CT scanner (Somatom Sensation, Siemens). The series were acquired at maximum inspiration before (unenhanced phase) and 30 seconds (hepatic arterial phase), 70 seconds (portal venous phase), and 300 seconds (equilibrium) after intravenous injection (3-4 ml/s) of $100 \mathrm{ml}$ of a low-osmolar, nonionic contrast agent with an iodine concentration of $300 \mathrm{mg} / \mathrm{ml}$ (Ultravist-300 Iopromide; Bayer Pharma AG, Berlin, Germany), using an injection pump through an 18-g cannula in the left or right antecubital vein. Scanning parameters were $120 \mathrm{kV}$ and $180 \mathrm{mAs}$ with dose modulation at a slice collimation of $4 \times 2.5$, rotation time of 0.5 seconds, and pitch of 1.25 for the 4-slice multi-detector CT scanner, and collimation of $32 \times 0.6 \mathrm{~mm}$, rotation time of 0.33 seconds, and pitch of 0.75 for the 64 -slice multi-detector CT. All series were reconstructed in 5-mm contiguous axial and coronal slices.

In our hospital, 4-phase CT of the upper abdomen was routinely performed for all patients who had no recent CT scan (acquired maximum 4 weeks before surgery) and for all atypical liver lesions on recent portal-venous-phaseonly scans from other hospitals (such as hypervascular or cystic-appearing liver metastases). If necessary, gadolinium-enhanced liver magnetic resonance imaging (MRI) was used as a problem solver. Several patients already underwent a preoperative CT scan at an outside institution. All of these images were reviewed by our radiologists and if needed additional scans were made.

All patients underwent IOUS (Prosound Alpha10; 10.0$\mathrm{MHz}$ linear intraoperative probe and 5.0/1.25-MHz convex probe, Aloka, Tokyo, Japan) performed by an experienced interventional radiologist, who carefully noted the exact size (maximum diameter), number, and location of all CRLM.

The intraoperative findings were saved. Data recorded included the number and size of hepatic malignant lesions and evidence of local extension of disease. The findings on preoperative and intraoperative imaging and surgical exploration were compared regarding number and extension of the hepatic lesions. To assess whether treatment was altered, all surgeons' reports were reviewed and evaluated. Ultimately, the surgical treatment strategy resection-radiofrequency ablation (RFA) alone or combined with resection-was based on the intraoperative findings.

\section{Results}

Between January 2007 and January 2009, 100 consecutive patients (55 men, 45 women) with CRLM underwent 117 surgical procedures, resection, RFA, or a combination of both. The characteristics of the patients and their primary tumors are summarized in Table 1 . The surgical treatment methods are summarized in Table 2.

Overall, intraoperative findings (IOUS plus surgical exploration) yielded additional information on number, size, localization, and/or local extension of metastases in 45 cases (Fig. 1).

In 38 of the patients, findings on IOUS differed from those of the preoperative CT scan. In 23 of those cases, IOUS identified more hepatic malignant lesions than the preoperative CT scan: 20 true new lesions, and 3 satellite lesions originating from known metastases. In four patients, IOUS detected fewer lesions. In one patient, the metastatic lesion was smaller on IOUS than anticipated on the preoperative CT scan, changing the surgical treatment to a less extensive resection.

Additional information on the localization of the hepatic lesions was gathered by IOUS and changed the surgical treatment in ten cases. This additional

Table 1 Characteristics of patients and localization of primary carcinomas

\begin{tabular}{ll}
\hline Characteristic & Data \\
\hline Total no. patients & 100 \\
Male/female ratio & $55 / 45$ \\
Age (yr) & \\
Median & 65 \\
Range & $37-82$ \\
Primary tumor & \\
Colon & 68 \\
Rectosigmoid & 11 \\
Rectum & 21 \\
\hline
\end{tabular}


Table 2 Summary of 117 surgical treatments of hepatic metastases in 100 patients

\begin{tabular}{lc}
\hline Surgical treatment & Frequency (\%) \\
\hline Hemihepatectomy & $23(20 \%)$ \\
Left & $12(10 \%)$ \\
Right & $9(8 \%)$ \\
Extended & $2(2 \%)$ \\
Partial resection $^{\mathrm{a}}$ & $46(39 \%)$ \\
RFA alone $_{\text {Combined treatment }}{ }^{\mathrm{b}}$ & $30(26 \%)$ \\
RFA and resection & $13(11 \%)$ \\
RFA and right portal vein ligation $^{\mathrm{c}}$ & $11(9 \%)$ \\
None $^{\mathrm{c}}$ & $2(2 \%)$ \\
Total & $5(4 \%)$ \\
\hline
\end{tabular}

${ }^{\text {a }}$ Partial resection comprises metastasectomies and segmentectomies

b RFA and right portal ligation was combined in two patients with bilobar liver metastases to achieve hypertrophy of the left liver segments, making a secondary hemihepatectomy possible. In one patient, portal vein ligation alone was attempted to achieve the same, but this patient developed extrahepatic metastatic disease

${ }^{c}$ No surgical treatment was undertaken in five patients because of diffuse intrahepatic metastases in three and peritoneal carcinomatosis in two patients. However, one patient with diffuse metastases underwent combined RFA and resection of his bilobar metastases 7 months after his explorative laparotomy and chemotherapy treatment information led to more extensive resection in two cases. In seven patients, a RFA procedure was performed instead or additionally. In one patient, the hepatic lesion was found to be located more superficial than expected, changing the preoperative plan to perform a RFA procedure into a wedge resection.

Cohen's kappa measure of concordance between CT and IOUS findings was 0.84 for the number of CRLM and 0.79 for number, exact size, and localization of CRLM.

Surgical exploration identified more extensive extrahepatic disease in seven patients. In four patients, exploration detected diaphragmatic invasion, which altered the treatment; partial diaphragmatic resection was performed en bloc with the liver metastasis.

In three patients, surgical exploration identified peritoneal metastatic deposits. In two of these patients, the metastatic deposits were so extensive that resection was not possible. However, the other patient had only limited extrahepatic disease (solitary peritoneal deposits), amenable for resection.

Intraoperative findings (IOUS plus surgical exploration) changed the preoperative surgical plan for 42 of 117 procedures. In 45 patients, the intraoperative findings differed from the information gathered preoperatively on CT; in only three patients, these discrepancies did not result in a change of treatment strategy.
Fig. 1 Concordance between pre- and intraoperative

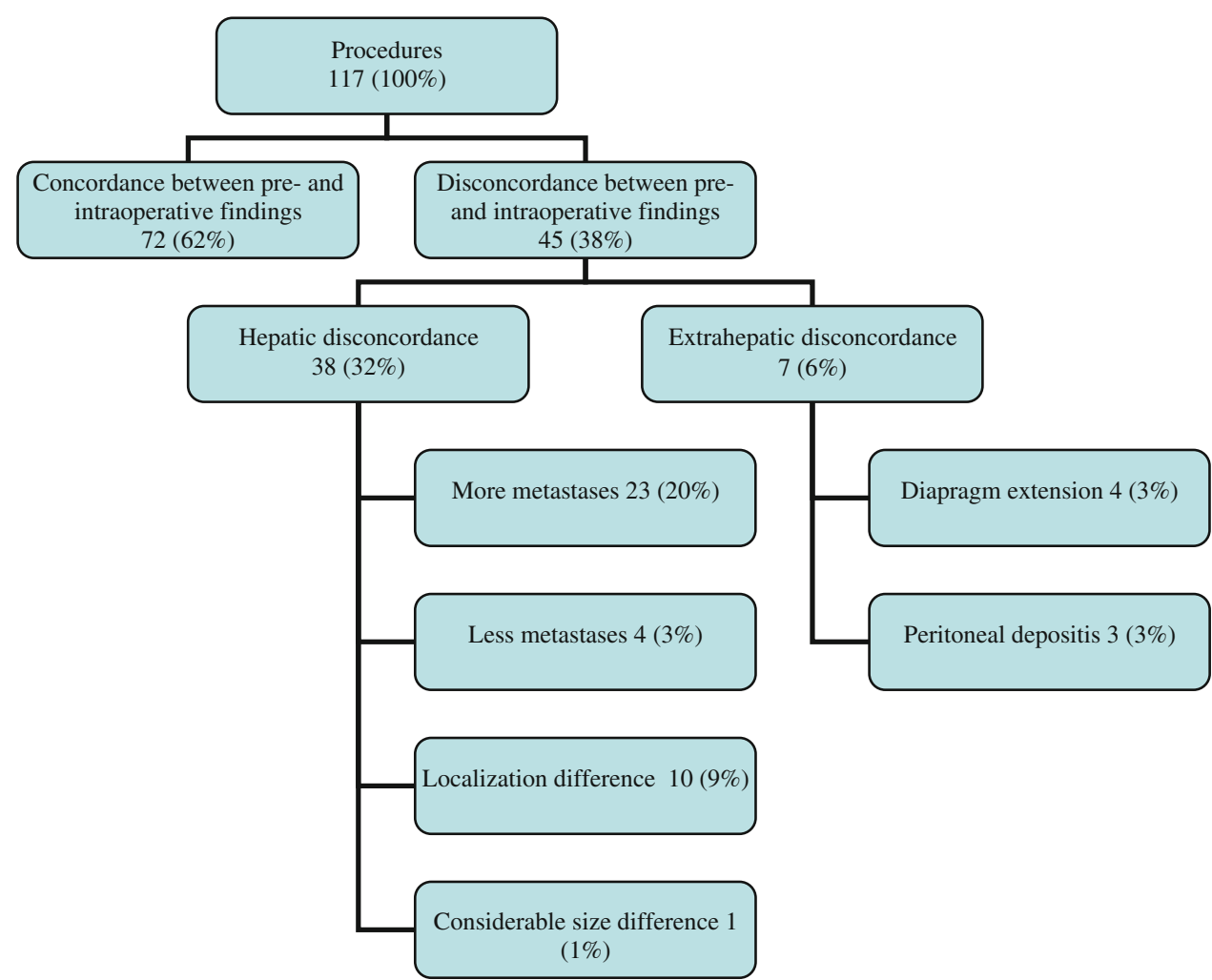


IOUS alone altered the surgical strategy 35 times during 117 treatments (30\%). In nearly all (92\%) cases, discrepancy between the preoperative CT scan and IOUS resulted in a change of surgical treatment. In general, IOUS identified more hepatic metastases, necessitating more extensive hepatic resection, RFA instead of resection, or a combination of those treatment modalities.

No surgical treatment was undertaken initially for an additional three patients because of diffuse intrahepatic metastases. However, one of the patients with diffuse metastases underwent combined treatment of RFA and resection 7 months after successful downstaging with chemotherapy.

\section{Discussion}

Intraoperative ultrasonography has been considered the "gold standard" since it was shown that the additional information found for many patients had a great impact on the surgical plan. Various authors reported that IOUS provided new information that influenced surgery in up to $50 \%$ of cases [1-4]. With the availability of modern imaging techniques, some have questioned the need for IOUS as diagnostic tool and only use it to plan their resection $[5,6]$.

In our series, however, the importance of IOUS in detecting preoperatively unknown lesions remains high. In $38 \%$ of patients, IOUS differed from the preoperative CT scan; more importantly, if discrepancies were found between IOUS and CT it nearly always changed the type of resection that was performed. These results are confirmed by others. Even though Ellsmere et al. questioned, "Why are we still performing IOUS during planned liver resection," they found that the frequency of unrecognized tumors during surgery did not change during the past 10 years. They compared 50 consecutive cases with a comparable historical group. They found that the rate of undetected tumors ranged between 14-20\%, changing their surgical plan in one fifth of patients [5].

In resent years not only the CT scan has been used for the preoperative workup. FDG-PET is of great importance in staging colorectal liver metastases, but whether FDGPET changes the "gold standard" of IOUS remains to be seen. Patients described in this series were for a large part also included in a Dutch national study in which patients were randomized between workup and follow-up with or without FDG-PET. As a result, half of the patients were analyzed and operated without preoperative PET scan. Therefore, we could not use these data in this analysis. The results of this study were presented recently and showed that workup with FDG-PET reduced the number of futile laparotomies; however, it did not influence disease-free survival or the total costs. The complete results will be published in the near future. Wiering et al. analyzed the accuracy of CT and FDG-PET compared with IOUS. Both PET and CT were sensitive for detecting lesions $>2 \mathrm{~cm}$. However, sensitivity declined rapidly when lesions were $<2 \mathrm{~cm}$. Only $16 \%$ of lesions $<1 \mathrm{~cm}$ were detected preoperatively [7]. The results of this study are comparable with our data that $30 \%$ more lesions were found during laparotomy. Strangely, this difference with preoperative knowledge had hardly any influence on clinical management. Previous studies from this group did show that FDGPET decreased the number of futile laparotomies by better detection of extrahepatic disease [8, 9].

Wildi et al. studied preoperative PET/CT with or without IOUS and correlated these results with histopathological and clinical follow-up data. They found that sensitivity of PET/CT alone was $63 \%$ versus $93 \%$ when $\mathrm{PET} / \mathrm{CT}$ was combined with IOUS. After preoperative chemotherapy, these data were comparable: PET/CT 77\% and combined with IOUS $100 \%$. In $35 \%$ of the cases, IOUS changed the surgical plan [10].

Rohren et al. also studied the importance of FDG-PET and compared this with IOUS. They found that FDG-PET is highly accurate in determining the presence or absence of hepatic metastases; however, FDG-PET is less accurate in determining the precise number and distribution of hepatic metastases. FDG-PET accurately defined the number and location on lesions in $55 \%$ of patients. As in other studies, lesion size was a significant factor in the detection of hepatic metastases by FDG-PET. Whether the preoperative plan based on PET changed due to intraoperative finding was not discussed [11].

In contrast with these data are the resent results of Figueras et al., which report a low sensitivity of CT scan alone; however, IOUS only changed their surgical procedure in $11 \%$ [12]. Overall, the conclusion can be made that IOUS remains the "gold standard" for the number and location of liver metastases. PET/CT is simply not sensitive enough to detect smaller lesions.

In our clinic, we hold the policy to spare as much liver parenchyma as possible-of course never at the cost of irradicality. There are two reasons for this policy. First, it reduces the need for major hepatectomies, which are associated with morbidity and mortality. Second, it makes secondary surgery for recurrent disease easier. IOUS helps us plan our resections. The varying results of whether IOUS changes the surgical plan depend, of course, mainly on the extent of the planned resection [13]. If you are sparing liver volume, it will change your surgical plan.

Even though IOUS can currently be considered the best there is, it is obviously not good enough. Almost half of the patients will have recurrent disease in the liver; most of these lesions develop out of small metastases present 
during the first exploration and therefore were missed by IOUS.

Various imaging techniques are analyzed to see whether preoperative accuracy can be improved.

Recently, we published the preliminary results of totalliver-volume perfusion CT (CTP) technique for the detection and characterization of liver metastases [14]. Twenty patients underwent helical $\mathrm{CT}$ of the total liver volume before and 11 times after intravenous contrast-material injection. To decrease distortion artefacts, all phases were coregistered using 3-D image fusion before creating bloodflow maps. Lesion-based sensitivity and specificity for liver metastases of first the conventional 4 phases and later all 12 phases, including blood-flow maps, were determined compared to intraoperative ultrasound and surgical exploration. These preliminary results showed significantly increased sensitivity of total-liver-volume CTP.

Currently, we also are using contrast-enhanced intraoperative ultrasound (CE-IOUS) to improve the results; however, it is too early to report our data.

Leen et al. recently showed that CE-IOUS was significantly more sensitive than IOUS (96.1\% versus $81.5 \%$ ) [15]. It altered their surgical management in almost $30 \%$ of cases. However, in this study there was no difference between preoperative imaging and conventional IOUS, which is somewhat surprising considering the current literature. Furthermore, the median size of the additional lesions found with CE-IOUS was $8 \mathrm{~mm}$, which is well over the detection limit of conventional IOUS. Even though the improved sensitivity of contrast-enhanced ultrasound is confirmed in some studies, others question its value [16, 17].

The current results of gadolinium-enhanced MRI for colorectal liver metastases are still under debate; however, sensitivity and specificity seems slightly higher compared with portal-venous-phase contrast-enhanced helical CT [18].

In conclusion, despite improvement of preoperative imaging technology, the intraoperative use of ultrasonography remains crucially important. The detection of preoperatively unknown lesions is high with great consequence on surgical therapy. New techniques, such as total-liver-volume perfusion $\mathrm{CT}$ and contrast-enhanced intraoperative ultrasound and MRI, are being studied to improve diagnostic accuracy of preoperative imaging.

Disclosures Dr. C. Sietses, Dr. M. R. Meijerink, Prof. Dr. S. Meijer, and Dr. M. P. van den Tol have no conflicts of interest or financial ties to disclose.

Open Access This article is distributed under the terms of the Creative Commons Attribution Noncommercial License which permits any noncommercial use, distribution, and reproduction in any medium, provided the original author(s) and source are credited.

\section{References}

1. Meijer S, Paul MA, Cuesta MA, Blomjous J (1995) Intra-operative ultrasound in detection of liver metastases. Eur J Cancer 31A(7-8):1210-1211

2. Bismuth H, Castaing D, Garden OJ (1987) The use of operative ultrasound in surgery of primary liver tumors. World J Surg 11(5):610-614

3. Zacherl J, Scheuba C, Imhof M, Zacherl M, Längle F, Pokieser P, Wrba F, Wenzl E, Mühlbacher F, Jakesz R, Steininger R (2002) Current value of intraoperative sonography during surgery for hepatic neoplasms. World J Surg 26(5):550-554

4. Parker GA, Lawrence W Jr, Horsley JS 3rd, Neifeld JP, Cook D, Walsh J, Brewer W, Koretz MJ (1989) Intraoperative ultrasound of the liver affects operative decision making. Ann Surg 209(5):569-577

5. Ellsmere J, Kane R, Grinbaum R, Edwards M, Schneider B, Jones D (2007) Intraoperative ultrasonography during planned liver resections: why are we still performing it? Surg Endosc 21(8):1280-1283

6. Rydzewski B, Dehdashti F, Gordon BA, Teefey SA, Strasberg SM, Siegel BA (2002) Usefulness of intraoperative sonography for revealing hepatic metastases from colorectal cancer in patients selected for surgery after undergoing FDG PET. AJR Am J Roentgenol 178(2):353-358

7. Wiering B, Ruers TJ, Krabbe PF, Dekker HM, Oyen WJ (2007) Comparison of multiphase CT, FDG-PET and intra-operative ultrasound in patients with colorectal liver metastases selected for surgery. Ann Surg Oncol 14(2):818-826

8. Wiering B, Krabbe PF, Dekker HM, Oyen WJ, Ruers TJ (2007) The role of FDG-PET in the selection of patients with colorectal liver metastases. Ann Surg Oncol 14(2):771-779

9. Ruers TJ, Langenhoff BS, Neeleman N, Jager GJ, Strijk S, Wobbes T, Corstens FH, Oyen WJ (2002) Value of positron emission tomography with [F-18]fluorodeoxyglucose in patients with colorectal liver metastases: a prospective study. J Clin Oncol 20(2):388-395

10. Wildi SM, Gubler C, Hany T, Petrowsky H, Clavien PA, Jochum W, Gerlach T, Fried M, Mullhaupt B (2008) Intraoperative sonography in patients with colorectal cancer and resectable liver metastases on preoperative FDG-PET-CT. J Clin Ultrasound 36(1):20-26

11. Rohren EM, Paulson EK, Hagge R, Wong TZ, Killius J, Clavien PA, Nelson RC (2002) The role of F-18 FDG positron emission tomography in preoperative assessment of the liver in patients being considered for curative resection of hepatic metastases from colorectal cancer. Clin Nucl Med 27(8):550-555

12. Figueras J, Planellas P, Albiol M, López-Ben S, Soriano J, Codina-Barreras A, Pardina B, Rodríguez-Hermosa JI, Falgueras L, Ortiz R, Maroto A, Codina-Cazador A (2008) Role of intraoperative echography and computed tomography with multiple detectors in the surgery of hepatic metastases: a prospective study. Cir Esp 83(3):134-138

13. Torzilli G, Procopio F, Botea F, Marconi M, Del Fabbro D, Donadon M, Palmisano A, Spinelli A, Montorsi M (2009) Onestage ultrasonographically guided hepatectomy for multiple bilobar colorectal metastases: a feasible and effective alternative to the 2-stage approach. Surgery 146:60-71

14. Meijerink MR, van Waesberghe JH, van der Weide L, van den Tol P, Meijer S, van Kuijk C (2008) Total-liver-volume perfusion CT using 3-D image fusion to improve detection and characterization of liver metastases. Eur Radiol 18:2345-2354

15. Leen E, Ceccotti P, Moug SJ, Glen P, MacQuarrie J, Angerson WJ, Albrecht T, Hohmann J, Oldenburg A, Ritz JP, Horgan PG (2006) Potential value of contrast-enhanced intraoperative 
ultrasonography during partial hepatectomy for metastases: an essential investigation before resection? Ann Surg 243(2):236240

16. Fioole B, de Haas RJ, Wicherts DA, Elias SG, Scheffers JM, van Hillegersberg R, van Leeuwen MS, Borel Rinkes IH (2007) Additional value of contrast enhanced intraoperative ultrasound for colorectal liver metastases. Eur J Radiol 27

17. Larsen LP, Rosenkilde M, Christensen H, Bang N, Bolvig L, Christiansen T, Laurberg S (2007) The value of contrast enhanced ultrasonography in detection of liver metastases from colorectal cancer: a prospective double-blinded study. Eur $\mathbf{J}$ Radiol 62(2):302-307

18. Bipat S, van Leeuwen MS, Comans EF, Pijl ME, Bossuyt PM, Zwinderman AH, Stoker J (2005) Colorectal liver metastases: CT, MR imaging, and PET for diagnosis-a meta-analysis. Radiology 237(1):123-131 